\title{
The influence of granular materials on the operation and membrane fouling characteristics of submerged MBRs
}

\author{
Tokihiro Kurita $^{\mathrm{a}}$, Katsuki Kimura ${ }^{\mathrm{a}}$, Yoshimasa Watanabe \\ ${ }^{a}$ Division of Environmental Engineering, Hokkaido University, N13W8, Kita-ku, Sapporo 060-8628, \\ Japan \\ ${ }^{\mathrm{b}}$ Center for Environmental Nano and Bio Engineering in Hokkaido University, N13W8, Kita-ku, \\ Sapporo 060-8628, Japan \\ Tel: +81-(0)11-706-6271, E-mail: toki@ec.hokudai.ac.jp, kkatsu@eng.hokudai.ac.jp
}

\begin{abstract}
Aeration to mitigate membrane fouling accounts for the largest fraction of energy consumed in membrane bioreactors (MBRs). To reduce the energy consumed during MBR operation, alternative methods must be developed to control membrane fouling. Granules should be introduced into submerged MBRs to mitigate membrane fouling due to their mechanical cleaning effect on the membrane surface. This study assessed the possible reduction in aeration for submerged MBRs through introducing granules and investigated membrane fouling characteristics from using granules. Two bench-scale MBRs operated in parallel with synthetic wastewater, and granules were introduced into one MBR. The MBR with granules operated stably even after aeration was reduced by $50 \%$. Reversible fouling was almost entirely controlled through introducing granules. However, the granules promoted irreversible fouling due to cake elimination, which acted as a dynamic filter. The overall benefit from granules was apparent; although, reversible fouling dominated in the absence of granules. The
\end{abstract}


foulant characteristics differed with granule use. These characteristic changes were also explained by the absence of the dynamic filtration effect.

\section{Introduction}

Although membrane bioreactors (MBRs) produce a high-quality effluent that is suitable for a variety of water reuse applications [1], they are still not mainstream technology for municipal wastewater treatment. One of the largest obstacles for widespread application of MBRs is high-energy consumption during operation. It has been reported that the energy consumed while operating an MBR is two times higher than a conventional activated sludge process [2]. Aeration to mitigate membrane fouling accounts for the largest fraction of energy consumed in MBRs [3,4]. Aeration may explain up to $70 \%$ of the total energy consumed in MBRs $[5,6]$. To further reduce energy consumption during MBR operation, it is necessary to control membrane fouling through alternative methods. Introducing granular materials into MBRs might solve this problem.

Granular materials were used as biofilm carriers into MBRs, which was investigated to combine the advantages of biofilm and MBR processes [7-9]. Yang et al. [10] and Khan et al. [11] reported that this combined process improved the removal efficiency of the total nitrogen in MBR processes due to the biomass attached on the materials. On the other hand, certain researchers reported that introducing granules into submerged MBRs could mitigate membrane fouling due to their mechanical cleaning effect on the membrane surface [12-15]. Additionally, $\mathrm{Hu}$ et al. [16] reported that membrane fouling was mitigated by the biochemical 
effect from carriers on the characteristics of the sludge. It may be possible to substantially reduce aeration rate in submerged MBRs by introduction of granules. Improvement in efficiency of the operation of submerged MBRs by granules (e.g., the degree of possible reduction in aeration) have rarely been assessed quantitatively, although Pradan et al. [17] reported that the effect of introduction of granules was similar to that obtained by doubling the aeration rate in terms of mitigating membrane fouling. Regarding mechanisms of how granules mitigate membrane fouling in submerged MBRs, it was shown that granules were effective in reducing cake resistance $[12,15,18]$. Jin et al. [15] analysed the composition of cake that formed with the use of granules and demonstrated that the granules reduced the amount of biopolymers such as protein-like substances in the cake. In contrast, as for irreversible fouling that cannot be controlled by physical cleaning [19], it is thought that the effect of granular materials is limited $[12,15]$. Characteristics of foulants causing irreversible fouling are likely to change when granular materials are introduced in submerged MBRs. Information on this point is not available, although it is very important in selection of membranes. If different constituents are involved in fouling in the case of MBRs with granules, membranes that are different from those used in conventional MBRs should be selected.

The objective of this study was to investigate the effect of granular materials on membrane fouling mitigation and aeration reduction during operation of a bench-scale MBR. Differences in fouling characteristics due to the granules, focusing on irreversible fouling that cannot be controlled by granules, were also examined. In this study, two identical bench-scale MBRs were operated by side by side with synthetic wastewater, and granules were introduced into 
one MBR. The potential aeration reduction from introducing granules was assessed through critical flux measurements. The membrane fouling characteristics were investigated through autopsies of the fouled membranes and analyses of the foulants extracted from the fouled membranes.

\section{Materials and methods}

\subsection{Operation of bench-scale MBRs}

Two identical bench-scale MBRs (volume: $8 \mathrm{~L}$ ) were operated in parallel, and granules were introduced into one MBR in this study. A schematic diagram of the experimental system is shown in Fig. S1 of the supporting information. Flat-sheet microfiltration membranes (Toray, Japan) composed of PVDF polymer were used in this study. The nominal pore size of the membranes was $0.1 \mu \mathrm{m}$. Aerators were placed under the membrane modules, and aeration was continuously performed (aeration rates: $17 \mathrm{~L} / \mathrm{min}$ ). Intermittent operation (1 min pause for every 12 min of operation) was performed. In this study, when membrane fouling became significant, the membrane modules were removed from the reactor. Next, the fouled membranes were manually wiped with a sponge and rinsed with tap water. The two MBRs were inoculated with sludge collected from an MBR that treats municipal wastewater at a municipal wastewater treatment facility (Soseigawa Wastewater Treatment Center, Sapporo, Japan). The temperature in the two MBRs was maintained at $20^{\circ} \mathrm{C}$ using a water bath.

The granular materials used in this study (BCN, Nisshinbo Chemical Inc., Japan) were originally developed as microorganism carriers for wastewater treatment and had a cylindrical 
shape. The granules' size (both diameter and height) and specific gravity were $4 \mathrm{~mm}$ and 1.01-1.15, respectively. The granules were composed of polyethylene glycol. At the apparent volume $0.8 \mathrm{~L}$ (corresponding to $10 \%$ of the reactor volume), the granules were placed into one MBR.

In this study, a short term experiment of approximately 2 weeks (Run 1) and a long-term experiment performed over 4 months (Run 2) were performed. Table 1 summarizes the MBR operational parameters in each Run. In Run 1, the aeration rate reduction through introducing granules into submerged MBRs was evaluated. The synthetic wastewater used in Run 1 had the same composition as in Kimura et al. [20]. Sodium acetate was the major carbon source in Run 1. After biomass acclimatization, the critical flux was measured using the improved flux step method proposed by van der Marel et al. [21]. The membrane flux was increased from 10.4 to $29.2 \mathrm{LMH}\left(0.25\right.$ to $\left.0.70 \mathrm{~m}^{3} / \mathrm{m}^{2} / \mathrm{d}\right)$ using steps of $2.08 \mathrm{LMH}\left(0.05 \mathrm{~m}^{3} / \mathrm{m}^{2} / \mathrm{d}\right)$. For each flux, filtration was performed for 15 minutes with a 5 minute pause. In Run 2, the membrane fouling characteristic changes caused by the granules were investigated. The synthetic wastewater used in Run 2 was prepared by following the guidelines proposed by the OECD [22]. The major carbon sources were peptone and meat extract in Run 2. Fouled membrane modules were removed from the reactor when the MBRs were operated for one month and four months to analyse the membrane fouling characteristics. To maintain the initial total membrane area, new membranes were then placed in the reactors. The filtration resistance of the fouled membranes was measured using a series of batch filtration experiments in a pressurized dead-end cell system. The applied pressure was $30 \mathrm{kPa}$ in the batch filtrations. The permeate flux was measured using an electronic balance, and the membrane filtration 
resistance was calculated using the following equations:

$R_{t}=\frac{\Delta P}{J \cdot \mu}$

$R_{t}=R_{m}+R_{r}+R_{i r}$

where $J$ is the membrane permeate flux $\left(\mathrm{m}^{3} / \mathrm{m}^{2} / \mathrm{s}\right), \Delta P$ is the trans-membrane pressure $(\mathrm{Pa}), \mu$ is the permeate viscosity $(\mathrm{Pa} \mathrm{s}), R_{t}$ is the total membrane filtration resistance $\left(\mathrm{m}^{-1}\right), R_{m}$ is the intrinsic membrane resistance $\left(\mathrm{m}^{-1}\right), R_{r}$ is the reversible membrane filtration resistance $\left(\mathrm{m}^{-1}\right)$, and $R_{i r}$ is the irreversible membrane filtration resistance $\left(\mathrm{m}^{-1}\right)$.

In this study, the foulants that caused physically irreversible fouling [19] were extracted and analysed. The fouled membranes were manually wiped with a sponge to remove the accumulated cake that accounted for the reversible fouling. Sodium hydroxide $(\mathrm{pH}=12)$ was used to extract the foulants from the fouled membranes. The membranes were soaked in the alkaline solution at $30^{\circ} \mathrm{C}$ for $24 \mathrm{~h}$.

\subsection{Analytical methods}

The concentrations of total organic carbon (TOC) and dissolved organic carbon (DOC) were determined using a TOC analyser (TOC-VCSH, Shimadzu, Japan). The dissolved oxygen (DO) concentration was measured using a DO meter (DO-24P, DKK-TOA, Japan). For the DOC analysis, the dissolved carbohydrates and dissolved proteins in the mixed liquor suspensions samples were filtered with a $0.45 \mu \mathrm{m}$ mix cellulose ester (MCE) filter (Advantec Tokyo, Japan). The phenol-sulfuric acid method [23] and Lowry method [24] were used to determine the carbohydrate and protein concentrations, respectively. Glucose and bovine serum albumin (BSA) were used as standards for measuring the carbohydrates and proteins, 
respectively. A fluorescence spectrophotometer equipped with a $150 \mathrm{~W}$ ozone-free xenon lump (RF-5300PC, Shimadzu, Japan) was used to measure the excitation-emission matrix (EEM) spectra. For the fluorescence measurements, the wavelengths for both emission and excitation were varied stepwise by $5 \mathrm{~nm}$.

In Run 2, the fouled membrane surface was observed through scanning electron microscopy (SEM) (S-4000, Hitachi, Japan). The fouled membranes were cut into $1 \mathrm{~cm}^{2}$ squares and immersed in $2 \%$ glutaraldehyde with $0.1 \mathrm{M}$ phosphate buffer $(\mathrm{pH}=7.2)$ at $5^{\circ} \mathrm{C}$ overnight to fix the fouled membrane surface morphology. The treated samples were then fixed with $1 \%$ osmium tetroxide with $0.1 \mathrm{M}$ phosphate buffer at room temperature for $2 \mathrm{~h}$. The fixed samples were dehydrated at a series of different concentrations. After dehydration, the samples were immersed twice in isoamyl acetate solution for $15 \mathrm{~min}$. The samples were dried using a critical point drying apparatus (HCP-1, Hitachi, Japan) to avoid deforming the fouled membrane surface morphology. The dried samples were coated with platinum to avoid accumulating static electric charge during the measurements.

\section{Results and discussion}

\subsection{Run 1}

\subsubsection{Critical flux}

The biomass in the two reactors was acclimated to the operational conditions during the preceding month. After acclimatizing the biomass, the critical fluxes in the two MBRs were measured. Fig. 1 shows the time course for the trans-membrane pressure (TMP) changes with 
stepwise membrane flux increases. When the MBR was operated without granules (aeration rate: $17 \mathrm{~L} / \mathrm{min}$ ), fluxes higher than 14.6 LMH rapidly increased the TMP, which implies that the critical flux under that condition was approximately 14.6 LMH. On the other hand, introducing granules into the reactor could increase the critical flux to approximately 20.8 LMH under the same aeration rate. Krause et al. [25] reported that introducing granules into a submerged MBR could increase the critical flux by approximately $30 \%$ because granules clean the membrane surface. In this study, the critical flux was increased by $>40 \%$ through using granules. Table 2 summarizes the dissolved organic component concentrations in mixed liquor suspensions and the MLSS concentration for the two MBRs in Run 1. For the MBR with granules, the MLSS concentration was higher than the other MBR operated without granules. However, the dissolved carbohydrate and protein concentrations were lower in the MBR with granules. The polysaccharides and proteins were assumed to be the major fractions that contribute to fouling [26]. The critical flux increase from using granules could be explained by both the cleaning effect on the membrane surface and the lower dissolved component concentrations in the mixed liquor suspensions.

The critical flux in the MBR operated with granules was also measured when aeration rate was reduced from 17 to $8.5 \mathrm{~L} / \mathrm{min}$. The TMP increase was comparable to observations from operations without granules but with a 2-fold greater aeration rate; this result suggests that introducing granules into an MBR reduces the aeration rate by $50 \%$. The DO concentrations after aeration rate reduction were sufficiently high $(>2 \mathrm{mg} / \mathrm{L})$. Based on the critical flux measurement results, continuous operation of the MBR with granules was attempted under the reduced aeration rate $(8.5 \mathrm{~L} / \mathrm{min})$, whereas in the MBR operated without granules, the 
aeration rate was doubled $(17 \mathrm{~L} / \mathrm{min})$.

\subsubsection{Aeration rate reduction through granule introduction}

Fig. 2 shows the time course for the TMP changes in Run 1. Sponge cleaning was performed at the time points indicated by arrows in Fig. 2. In Run 1, the MBR TMP without granules increased to $40 \mathrm{kPa}$ within 100 hours of operation. After physically cleaning the membrane surface, the TMP considerably decreased, but it continued to rapidly increase. On the other hand, the MBR with granules could be operated without the physical cleaning even with the reduced aeration rate (Fig. 2 (b)). Clearly, introducing granules into a submerged MBR could mitigate membrane fouling and reduce the aeration rate effectively.

\subsection{Run 2}

\subsubsection{The effect of introducing granules on long-term MBR operation}

Table 3 summarizes the dissolved organic component concentrations in the mixed liquor suspensions and the MLSS concentration in Run 2. The MLSS in the MBR with granules was higher than without granules, which was similar to Run 1. The concentration of dissolved carbohydrate in the MBR without granules was higher than with granules, whereas the dissolved protein concentrations were almost comparable for the two MBRs. Fig. 3 shows EEM fluorescence spectra for the dissolved components in the two MBR mixed liquor suspensions. The EEM fluorescence spectra measurements were repeatedly performed in Run 2, and all of the measurement results were similar. Representative spectra are shown in Fig. 3. A peak that can be attributed to a humic acid-like substance was observed at Ex/Em $=330$ $\mathrm{nm} / 400 \mathrm{~nm}$ [27], which dominated in both MBRs. It was suggested that the MBRs' dissolved 
organic matter characteristics were similar to a certain extent. It should be noted that the carbohydrates were not detected by the EEM analysis, though.

Fig. 4 shows the time course for the TMP changes in the two MBRs recorded in Run 2. Physical cleaning (wiping with a sponge) was performed at the time points indicated by arrows in Fig. 4. The TMP in the MBR operated without granules rapidly increased within 10 days. Physically cleaning the membrane surface substantially reduced the TMP, indicating that membrane fouling in the MBR without granules was mainly physically reversible fouling. However, a rapid TMP increase was repeatedly observed in the MBR without granules. In contrast, the MBR operated with granules could be operated for four months without physical cleaning, and the TMP increase was fairly slow. Clearly, introducing granules into the submerged MBRs was effective in mitigating membrane fouling for long-term operation.

Fig. 5 shows the SEM images of the membrane surface collected after one month of operation. The membrane surface was covered by accumulations in the MBR operated without granules (Fig. 5 (b)). On the other hand, in the MBR operated with granules, no cake accumulation was observed, and the surface morphology was almost the same as the virgin membrane (Fig. 5 (c)). The SEM observation clearly demonstrates that introducing granules into the submerged MBRs efficiently prevents cake accumulation on the membrane surfaces. Yang et al. [12] reported that accumulation of a gel layer on the surface of hollow fiber membranes occurred when they used granules with a submerged MBR. In this study, as stated above, no accumulation of cake or gel was observed. Membrane configurations might explain this difference. In the work of Yang et al. [12], hollow fiber membranes were used and access 
of granules to the membrane surface (particularly to the inside of membrane bundles) was probably limited. In contrast, in this study in which flat-sheet membranes were used, access of granules to the membrane surface was easy and more uniform, which might explain the high efficiency of cleaning observed in this study to some extent. However, the TMP in the MBR with granules increased gradually over the four months of operation; this TMP increase can be attributed to irreversible fouling, which cannot be restored by physical cleaning.

\subsubsection{The filtration resistance of the fouled membranes}

Table 4 summarizes the filtration resistance of the fouled membranes assessed at one month and four months of MBR operation. Reversible fouling dominated ( $>80 \%)$ in the MBR operated without granules. The level of irreversible fouling increased in both MBRs with operation time. The contribution of irreversible fouling to the total filtration resistance was significant for the MBR operated with granules. After four months of operation, the irreversible membrane filtration resistance in the MBR with granules was higher than without granules by $>2$-fold. Introducing granules effectively controlled reversible fouling, whereas the development of irreversible fouling was accelerated with granules. Fig. 6 shows SEM images of the fouled membrane surfaces collected at four months of operation after physical cleaning (sponge wiping). Sponge wiping could remove the accumulated cake from the membrane surface. For the MBR operated without granules, the membrane surface was clean after the sponge wiping (Fig. 6 (a)). In contrast, in the MBR operated with granules, certain components tightly adhered to the membrane surface; those components were also observed in other samples collected from different sections of the fouled membrane in the MBR operated with granules. The cake accumulated on the membrane surface can act as dynamic 
membrane that traps the particles in mixed liquor suspensions [28,29]. Lin et al. [30] reported that soluble microbial products (SMP) were removed by the membrane and membrane surface cake layer. In the MBR with granules, cake accumulation on the membrane was prevented (Fig. 5 (c)), whereas the foulants easily accessed the membrane surface and eventually caused irreversible fouling. Additionally, for the MBR with granules, the foulants characteristics that caused irreversible fouling were different, likely due to the absence of a membrane surface cake layer (details are discussed in the 3.2.3 Characteristics of the extracted foulants). The fouled membrane permeability could be restored to the level of the virgin membrane through chemical cleaning (soaking in $1000 \mathrm{ppm}$ of $\mathrm{NaOCl}$ solution at $30^{\circ} \mathrm{C}$ for $24 \mathrm{~h}$ ) regardless of whether granules introduced; this indicates that the irreversible fouling in this study was caused by organic components in the mixed liquor suspensions.

\subsubsection{Characteristics of the extracted foulants}

Table 5 summarizes the organic component levels extracted from the fouled membranes in Run 2. The extracted foulants levels increased with the operation time. The proportion of proteins in the foulants increased with time. The levels of carbohydrates extracted in both MBRs were comparable, whereas the levels of proteins for the operation with granules were higher than without granules. Fig. 7 shows EEM fluorescence spectra for the foulants extracted from the fouled membranes in Run 2. When the MBRs were operated for one month, the extracted foulants EEM fluorescence spectra were similar regardless of whether the granules were introduced (Fig. 7 (a), (b)). After four months of operation, the peak attributed to protein-like substances $(\mathrm{Ex} / \mathrm{Em}=270 \mathrm{~nm} / 320 \mathrm{~nm})$ dominated in both MBRs (Fig. 7 (c), (d)), which is consistent with the increase of proteins for the longer operation periods (Table 
5). As shown in Fig. 3, the dominant peak in the EEM fluorescence spectra for the dissolved components in the mixed liquor suspensions indicated humic acid-like substances. However, in the foulants EEM fluorescence spectra, the protein-like substances peak dominated, suggests that proteins accelerated irreversible fouling in the MBRs.

In the EEM fluorescence spectra for the foulants extracted from the MBR operated with granules, the humic acid-like substances peak also became significant upon operation for four months, which was not observed in the EEM fluorescence spectra for the MBR without granules (Fig. 7 (c), (d)). This observation implies that introducing granules into MBRs changed the foulants characteristics. As discussed previously, a dynamic filtration effect was not expected for the MBR operated with granules. As a result, the contribution of humic acid-like substances to irreversible fouling increased for the MBR operated with granules. Macro-molecules on the fouled membrane surface collected from the MBR operated with granules (Fig. 6 (b)) might have humic-acid characteristics; this has not been confirmed and is currently under investigation in our laboratory.

\section{Conclusions}

Introducing granules into a submerged MBR increased the critical flux by more than $40 \%$. Apparently, the MBR aeration rate can be reduced by using granules. In this study, through using granules, MBR operation was stable after aeration was reduced by $50 \%$. Reversible fouling was completely controlled by using the granules, whereas granules promoted irreversible fouling over long-term operation. The foulants characteristics differed upon use of 
the granules. The contribution of humic acid-like substances in foulants became significant in for MBRs operated with granules. These changes could be explained by the absence of a dynamic filtration effect on the membrane surface due to the mechanical cleaning effect of the granules. The results obtained in this study imply that different membrane should be selected when MBRs are operated with granules.

\section{Acknowledgements}

This research was financially supported by Core Research of Evolutional Science \& Technology (CREST) for "Innovative Technology and System for Sustainable Water Use" from the Japan Science and Technology Agency (JST).

\section{References}

[1] T. Asano, F.L. Burton, H.L. Leverenz, R. Tsuchihashi, G. Tchobanoglous, Water Reuse: Issues, Technologies, and Applications, McGraw Hill, New York, 2006.

[2] A. Fenu, J. Roels, T. Wambecq, K. De Gussem, C. Thoeye, G. De Gueldre, B. van De Steene, Energy audit of a full scale MBR system, Desalination 262 (2010) 121-128.

[3] J.A. Gil, L. Túa, A. Rueda, B. Montaño, M. Rodríguez, D. Prats, Monitoring and analysis of the energy cost of an MBR, Desalination 250 (2010) 997-1001.

[4] F. Fatone, P. Battistoni, P. Pavan, F. Cecchi, Operation and Maintenance of Full-Scale Municipal Membrane Biological Reactors: A Detailed Overview on a Case Study, Ind. Eng. Chem. Res. 46 (2007) 6688-6695. 
[5] M. Kraume, A. Drews, Membrane Bioreactors in Waste Water Treatment - Status and Trends, Chem. Eng. Technol. 33 (2010) 1251-1259.

[6] B. Verrchaht, T. Maere, I. Nopens, C. Brepols, S. Judd, The cost of a large-scale hollow fibre MBR, Water Res. 44 (2010) 5274-5283.

[7] H.-H. Ngo, W. Guo, W. Xing, Evaluation of novel sponge-submerged membrane bioreactor (SSMBR) for sustainable water reclamation, Bioresour. Technol. 99 (2008) $2429-2435$.

[8] W. Guo, H.-H. Ngo, F. Dharmawan, C.G. Palmer, Roles of polyurethane foam aerobic moving and fixed bed bioreactors, Bioresour. Technol. 101 (2010) 1435-1439.

[9] I. Ivanovic, T.O. Leiknes, The biofilm membrane bioreactor (BF-MBR) - a review, Desalin. Water Treat. 37 (2012) 288-295.

[10] S. Yang, F. Yang, Z. Fu, Lei, Comparison between a moving bed membrane bioreactor and a conventional membrane bioreactor on organic carbon and nitrogen removal, Bioresour. Technol. 100 (2009) 2369-2374.

[11] S.J. Khan, S. Ilyas, S. Javid, C. Visvanathan, V. Jegatheesan, Performance of suspended and attached growth MBR systems in treating high strength synthetic wastewater, Bioresour. Technol. 102 (2011) 5331-5336.

[12] Q. Yang, J. Chen, F. Zhang, Membrane fouling control in a submerged membrane bioreactor with porous, flexible suspended carriers, Desalination 189 (2006) 292-302.

[13] W.-N. Lee, I.-J. Kang, C.-H. Lee, Factors affecting filtration characteristics in membrane-coupled moving bed biofilm reactor, Water Res. 40 (2006) 1827-1835.

[14] B. Siembida, P. Cornel, S. Krause, B. Zimmermann, Effect of mechanical cleaning with granular material on the permeability of submerged membrane in the MBR process, Water 
Res. 44 (2010) 4037-4046.

[15] L. Jin, S.L. Ong, H.Y. Ng, Fouling control mechanism by suspended biofilm carriers addition in submerged ceramic membrane bioreactors. J. Membrane Sci. 427 (2013) 250-258.

[16] J. Hu, H. Ren, K. Xu, J. Geng, L. Ding, X. Yan, K. Li, Effect of carriers on sludge characteristics and mitigation of membrane fouling in attached-growth membrane bioreactor, Bioresour. Technol. 122 (2012) 35-41.

[17] M. Pradhan, S. Vigneswaran, J. Kandasamy, R.B. Aim, Combination effect of air and mechanical scouring of membrane for fouling reduction in submerged membrane reactor, Desalination 288 (2012) 58-65.

[18] X. Huang, C.-H. Wei, K.-C. Yu, Mechanism of membrane control by suspended carriers in a submerged membrane bioreactor, J. Memb. Sci. 309 (2009) 7-16.

[19] K. Kimura, Y. Hane, Y. Watanabe, G. Amy, N. Ohkuma, Irreversible membrane fouling during ultrafiltration of surface water, Water Res. 38 (2004) 3431-3441.

[20] K. Kimura, T. Naruse, Y. Watanabe, Changes in characteristics of soluble microbial products in membrane bioreactors associated with different solid retention times: Relation to membrane fouling, Water Res. 43 (2009) 1033-1039.

[21] P. van der Marel, A. Zwijnenburg, A. Kemperman, M. Wessling, H. Temmink, W. van der Meer, An improved flux-step method to determine the critical flux and the critical flux for irreversibility in a membrane bioreactor, J. Memb. Sci. 332 (2009) 24-29.

[22] OECD, OECD Guidelines for Testing Chemicals, Organization for Economic Cooperation and Development, Paris, Guideline 302A \& 303A, 1993.

[23] M. Dubois, K.A. Gilles, J.K. Hamilton, P.A. Rebers, F. Smith, Colorimetric method for determination of sugars and related substances, Anal. Chem. 28 (1956) 350-356. 
[24] O.H. Lowry, N.J. Rosebrough, A.L. Farr, R.J. Randall, Protein measurement with the folin phenol reagent, J. Biol. Chem. 193 (1951) 265-275.

[25] S. Krause, B. Zimmermann, U. Meyer-Blumenroth, W. Lamparter, B. Siembida, P. Cornel, Enhanced membrane bioreactor process without chemical cleaning, Water Sci. Technol. 61 (2010) 2575-2580.

[26] A. Drews, Membrane fouling in membrane bioreactors-characterisation, contradictions, cause and cures (review), J. Memb. Sci. 363 (2010) 1-28.

[27] W. Chen, P. Westerhoff, J.A. Leenheer, K. Boolsh, Fluorescence excitation-emission matrix regional integration to quantify spectra for dissolved organic matter, Environ. Sci. Technol. 37 (2003) 5701-5710.

[28] Y. Kiso, Y.-J. Jung, M.-S. Park, W. Wang, M. Shimase, T. Yamada, K.-S. Min, Coupling of sequencing batch reactor and mesh filtration: Operational parameters and wastewater treatment performance, Water Res. 39 (2005) 4887-4898.

[29] X. Zhang, Z. Wang, Z. Wu, F. Lu, J. Tong, L. Zang, Formation of dynamic membrane in an anaerobic membrane bioreactor for municipal wastewater treatment, Chem. Eng. J. 165 (2010) 175-183.

[30] H. Lin, B.-Q. Liao, J. Chen, W. Gao, L. Wang, F. Wang, X. Lu, New insights into membrane fouling in a submerged anaerobic membrane bioreactor based on characterization of cake sludge and bulk sludge, Bioresour. Technol. 102 (2011) 2373-2379. 


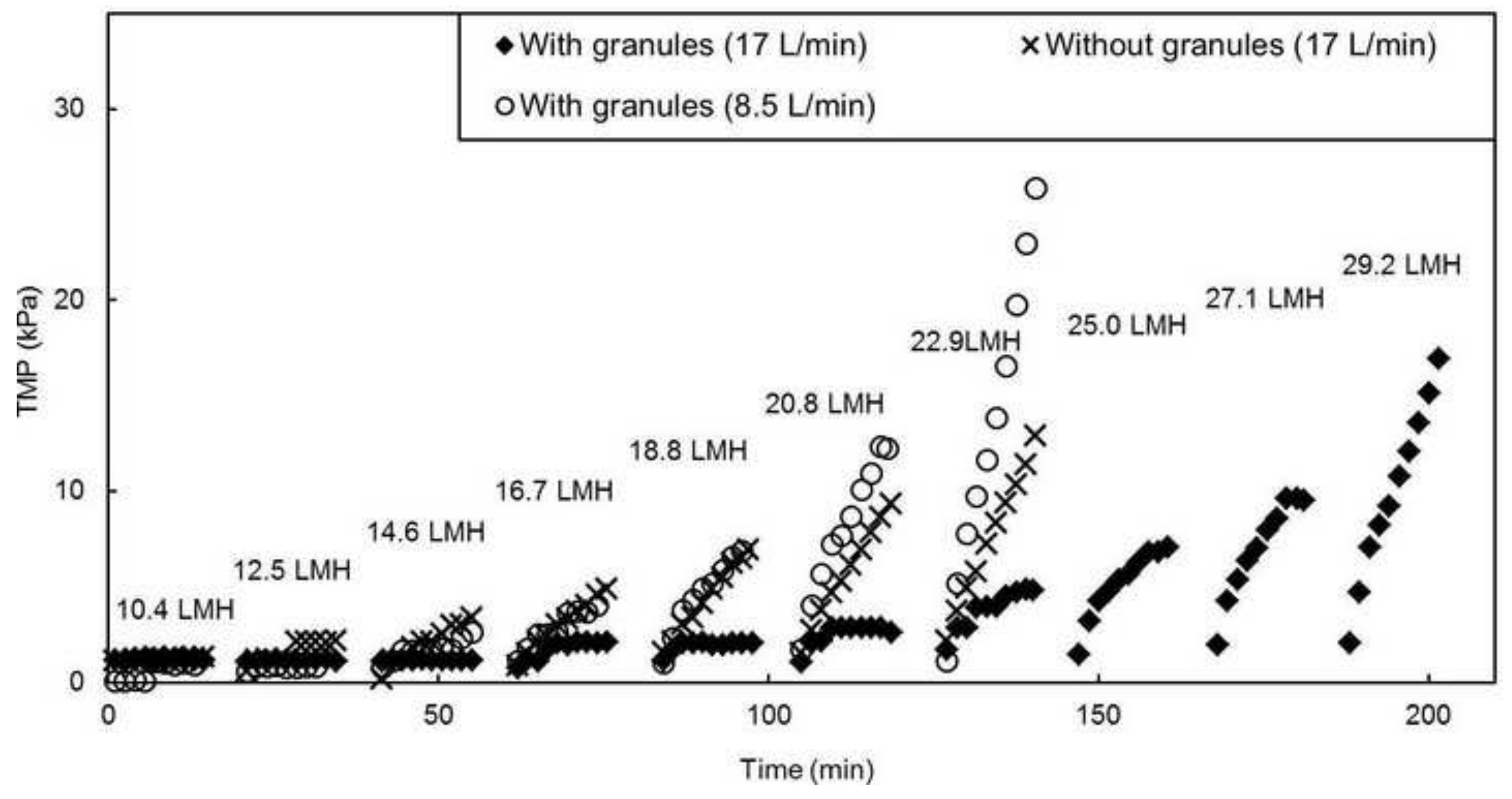



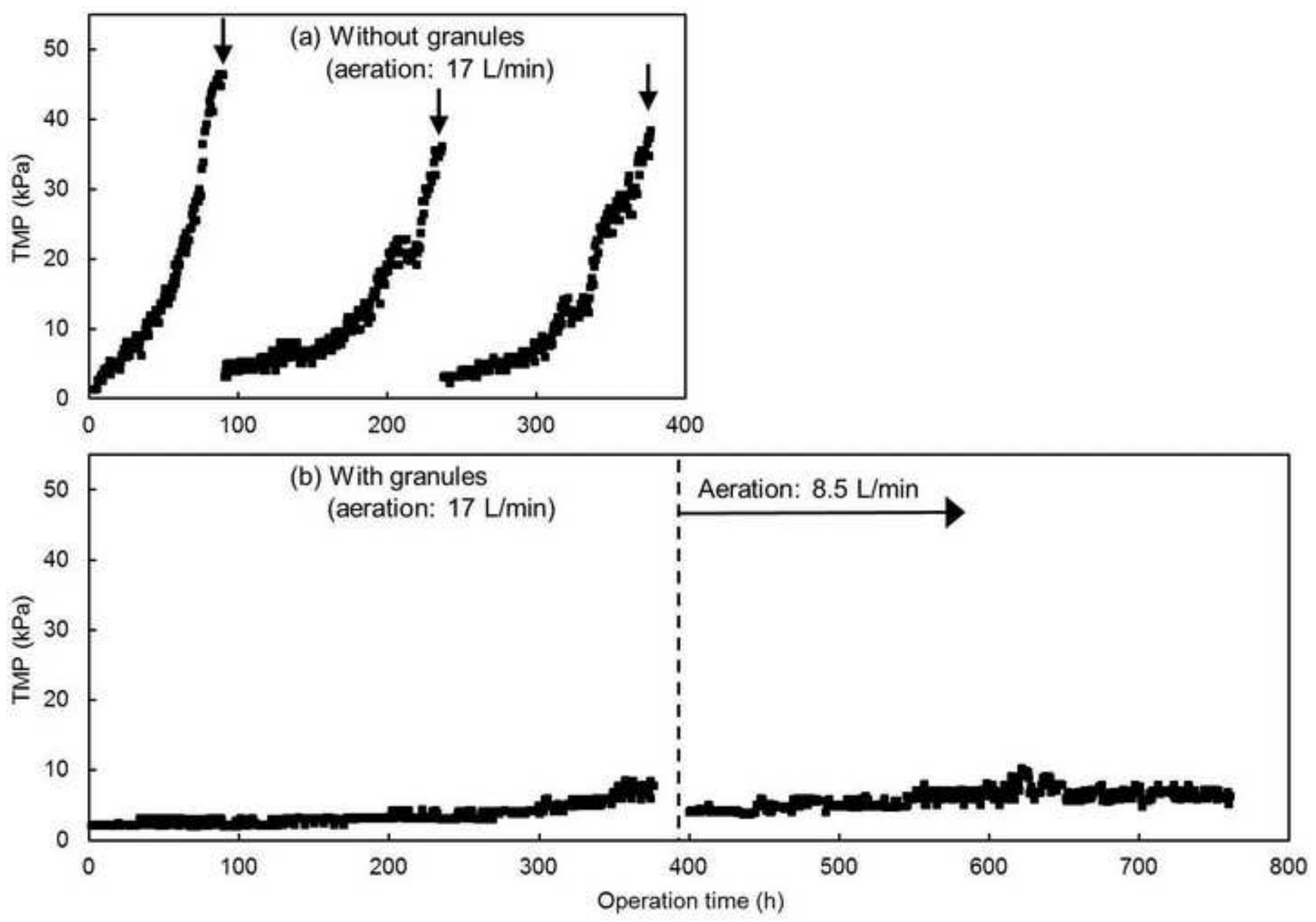

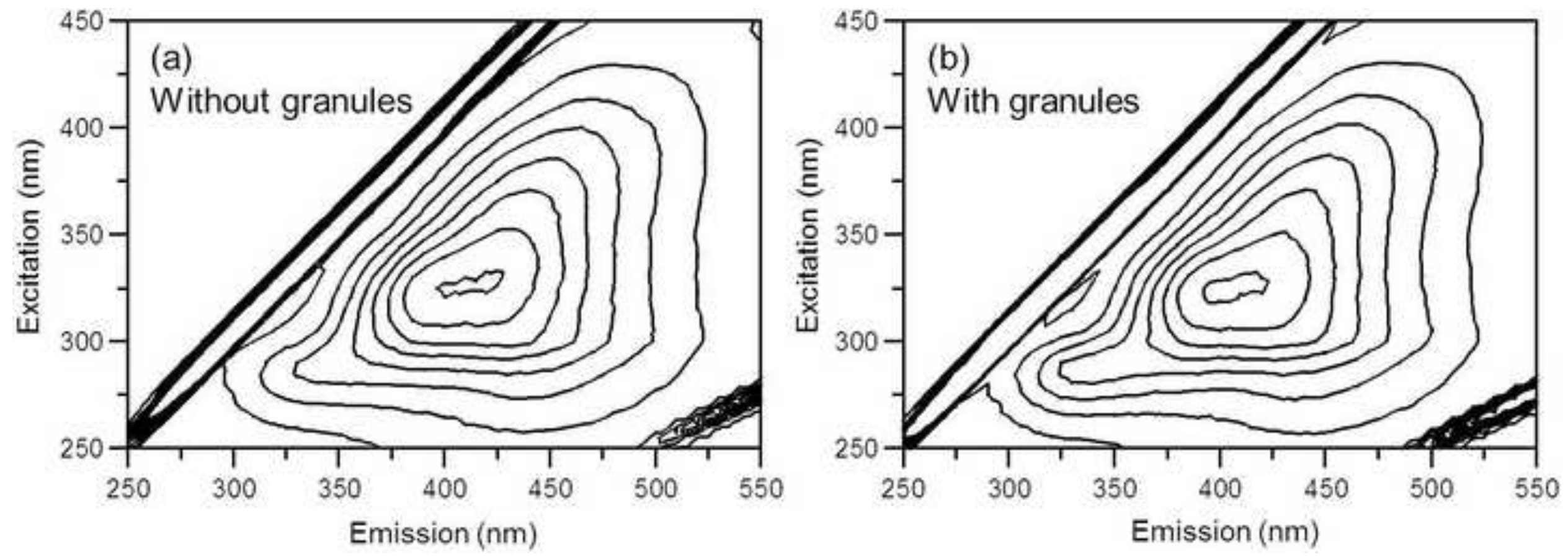

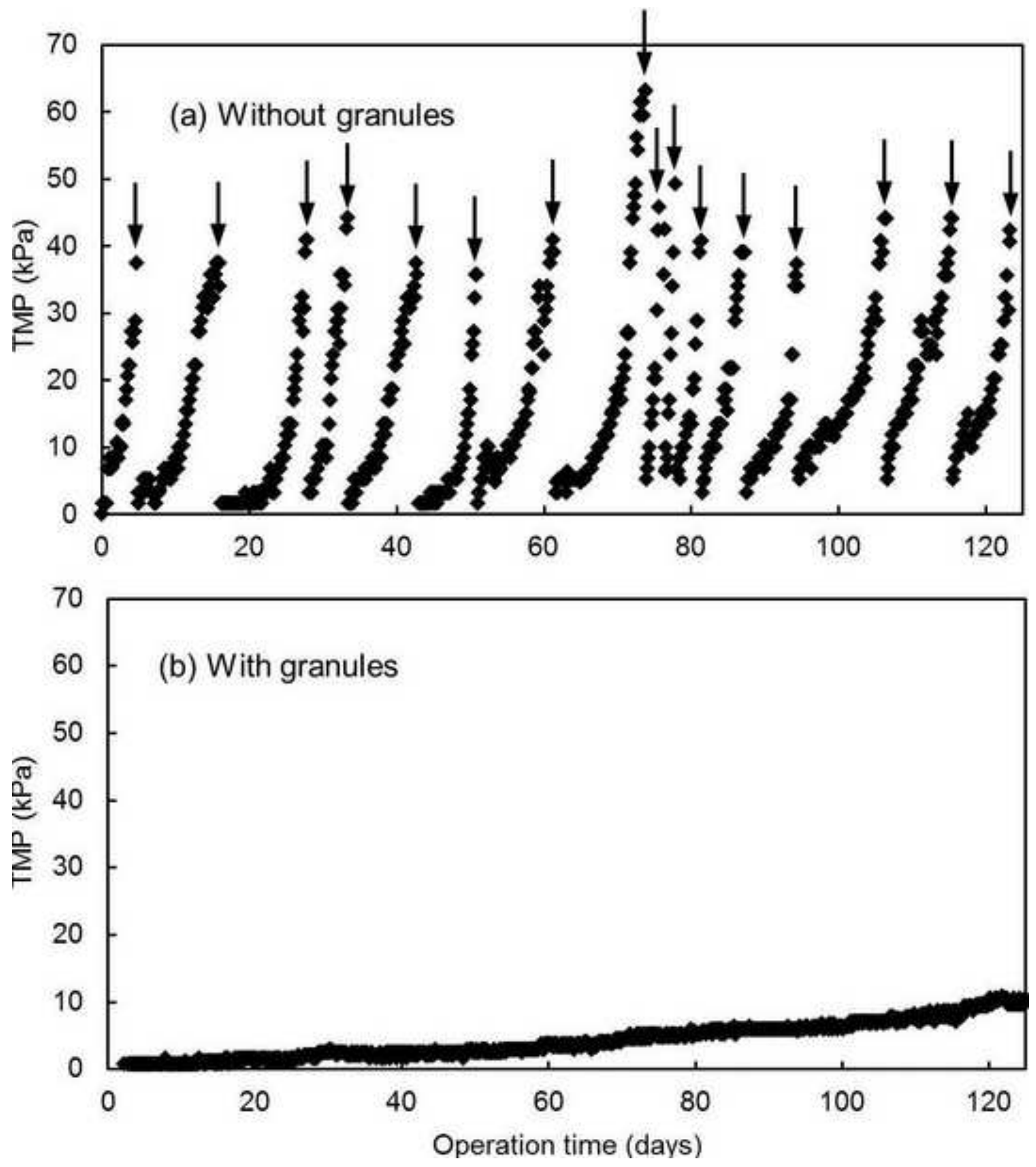

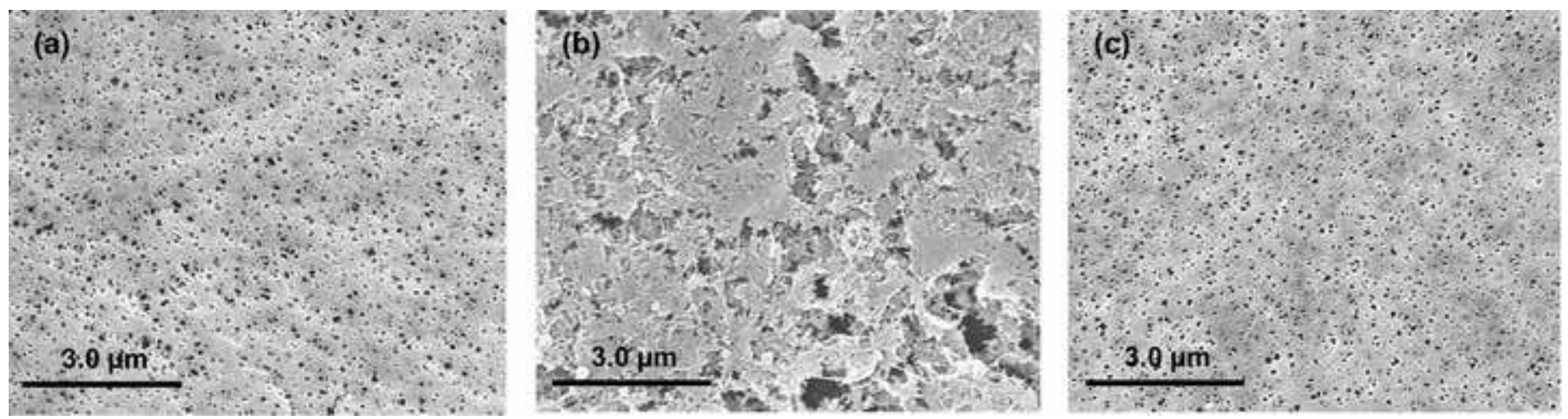
After 1 month
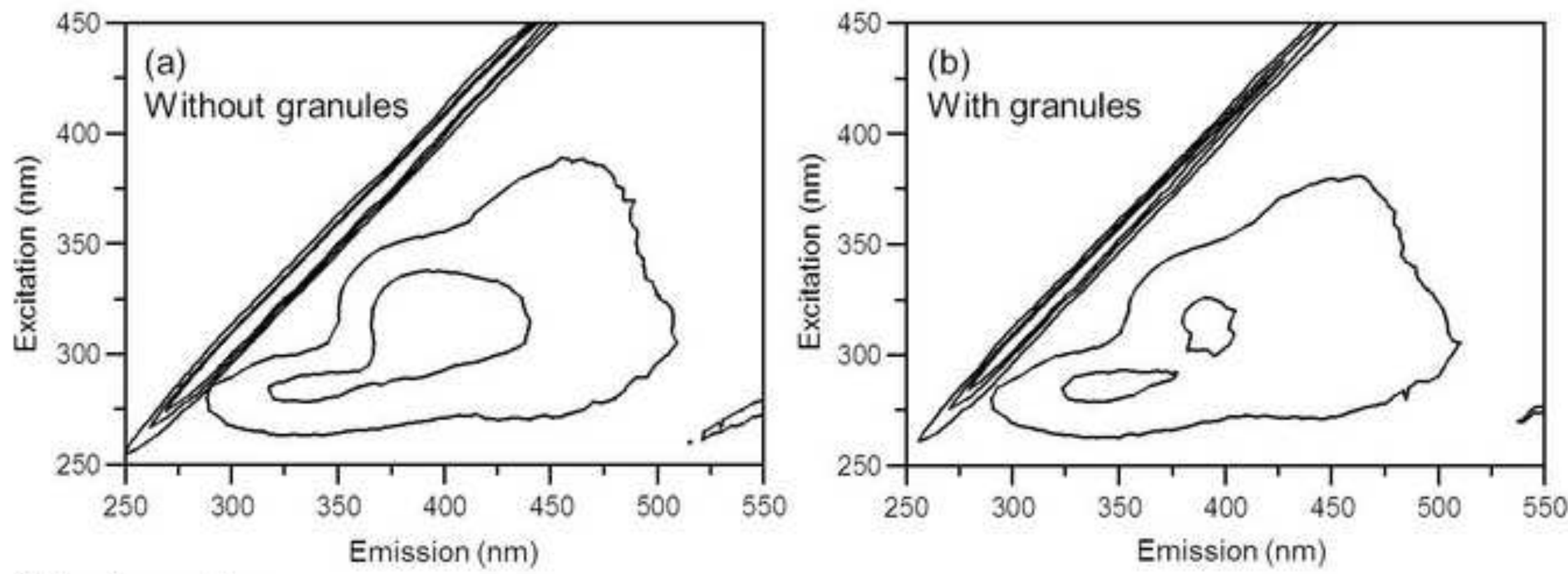

After 4 months
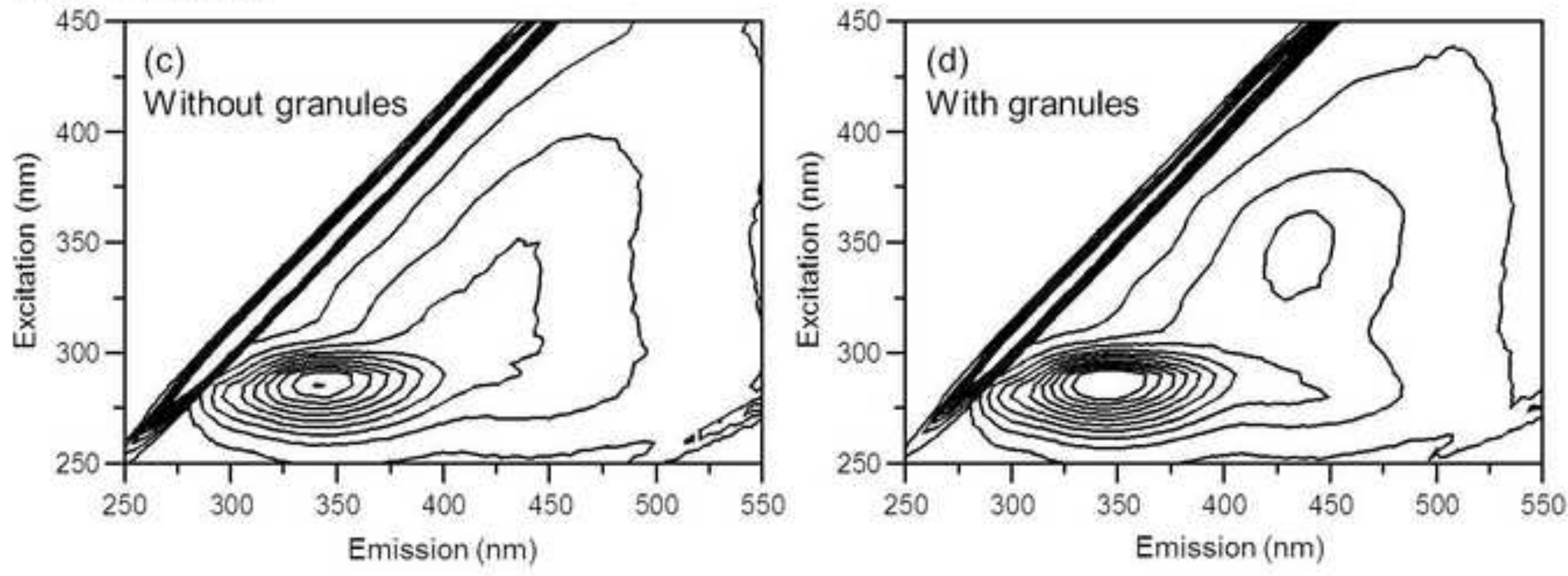
Table 1 Operational parameters of the two reactors.

Table 2 The dissolved organic component concentrations in the mixed liquor suspensions and MLSS in Run 1.

Table 3 The dissolved organic component concentrations in mixed liquor suspensions and MLSS in Run 2.

Table 4 The filtration resistances of the fouled membrane.

Table 5 The level of organic components in the extracted foulants in Run 2.

Fig. S1 The schematic diagram of the experimental system.

Fig. 1 The time course for the TMP changes in a stepwise increased membrane flux.

Fig. 2 The time course for the TMP changes in Run 1.

Fig. 3 EEM fluorescence spectra for the components dissolved in the mixed liquor suspension in Run 2.

Fig. 4 The time course for the TMP change in Run 2.

Fig. 5 SEM images of the membrane surface at one month of operation:

(a) Virgin membrane, (b) without granules, and (c) with granules. Physical cleaning was not performed prior to the SEM observations.

Fig. 6 SEM images of the membrane surface at four months of operation (after physical cleaning). (a) Without granules, and (b) with granules.

Fig. 7 EEM fluorescence spectra of the foulants in Run 2. 
Table 1

Reactor volume (L)

Membrane flux (LMH)

Membrane area $\left(\mathrm{m}^{2}\right)$

SRT (d)

HRT (h)
Run 1

8

$16.7\left(0.40 \mathrm{~m}^{3} / \mathrm{m}^{2} / \mathrm{d}\right)$

0.12

10

4.0
Run 2

7.5

$18.8\left(0.45 \mathrm{~m}^{3} / \mathrm{m}^{2} / \mathrm{d}\right)$

0.06

22

7.3 
Table 2

$\operatorname{MLSS}(\mathrm{mg} / \mathrm{L})$

Carbohydrate (mg/L)

Protein (mg/L)

C/P
Without granules

$2900 \pm 400$

$27.6 \pm 6.3$

$8.3 \pm 6.9$

3.3
With granules

$3300 \pm 100$

$12.7 \pm 4.9$

$9.6 \pm 6.0$

1.3 
Table 3

MLSS (mg/L)

Carbohydrate (mg/L)

Protein (mg/L)

C/P
Without granules

$5200 \pm 400$

$35.4 \pm 11.7$

$12.0 \pm 2.8$

3.0
With granules

$6600 \pm 700$

$26.8 \pm 19.1$

$11.7 \pm 2.8$

2.3 
After 1 month

\begin{tabular}{rcrcrcccr} 
& \multicolumn{2}{c}{ Without granules } & \multicolumn{2}{c}{ With granules } & \multicolumn{2}{c}{ Without granules } & \multicolumn{2}{c}{ With granules } \\
& $10^{11}\left(\mathrm{~m}^{-1}\right)$ & $\%$ & $10^{11}\left(\mathrm{~m}^{-1}\right)$ & $\%$ & $10^{11}\left(\mathrm{~m}^{-1}\right)$ & $\%$ & $10^{11}\left(\mathrm{~m}^{-1}\right)$ & $\%$ \\
$R_{m}$ & 0.6 & 10.5 & 0.6 & 31.6 & 0.6 & 0.8 & 0.6 & 3.9 \\
$R_{r}$ & 4.8 & 84.2 & 0.4 & 21.0 & 69.1 & 93.8 & 6.2 & 40.5 \\
$R_{i r}$ & 0.3 & 5.3 & 0.9 & 47.4 & 4.0 & 5.4 & 8.5 & 55.6 \\
$R_{t}$ & 5.7 & & 1.9 & & 73.7 & & 15.3
\end{tabular}


Table 5

After 1 month

Without granules With granules

TOC $\left(\mathrm{mg} / \mathrm{m}^{2}\right)$

Carbohydrate $\left(\mathrm{mg} / \mathrm{m}^{2}\right)$

Protein $\left(\mathrm{mg} / \mathrm{m}^{2}\right)$

C / P
140

89

160

0.56
120

75

210

0.36
After 4 months

Without granules With granules

220260

160

140

490

570

0.33

0.25 